\title{
Analysis of the dynamics and origin of epileptic activity in patients with tuberous sclerosis evaluated for surgery of epilepsy
}

\author{
Alberto J.R. Leal ${ }^{\mathrm{a}, \mathrm{b}, *}$, Ana I. Dias ${ }^{\mathrm{b}}$, José P. Vieira ${ }^{\mathrm{b}}$, Ana Moreira ${ }^{\mathrm{b}}$, Luís Távora ${ }^{\mathrm{c}}$, \\ Eulália Calado b \\ a Department of Neurophysiology, Hospital Júlio de Matos, Av ${ }^{a}$ do Brasil nr 53, 1749-002 Lisbon, Portugal \\ ${ }^{\mathrm{b}}$ Department of Pediatric Neurology, Hospital Dona Estefânia, Lisbon, Portugal \\ ${ }^{\mathrm{c}}$ Department of Pediatric Neurosurgery, Hospital Dona Estefânia, Lisbon, Portugal
}

Accepted 23 November 2007

\begin{abstract}
Objective: The epilepsies associated with the tuberous sclerosis complex (TSC) are very often refractory to medical therapy. Surgery for epilepsy is an effective alternative when the critical link between the localization of seizure onset in the scalp and a particular cortical tuber can be established. In this study we perform analysis of ictal and interictal EEG to improve such link.

Methods: The ictal and interictal recordings of four patients with TSC undergoing surgery for epilepsy were submitted to independent component analysis (ICA), followed by source analysis, using the sLORETA algorithm. The localizations obtained for the ictal EEG and for the average interictal spikes were compared.

Results: The ICA of ictal EEG produced consistent results in different events, and there was good agreement with the tubers that were successfully removed in three of the four patients (one patient refused surgery). In some patients there was a large discrepancy between the localization of ictal and interictal sources. The interictal activity produced more widespread source localizations.

Conclusions: The use of ICA of ictal EEG followed by the use of source analysis methods in four cases of epilepsy and TSC was able to localize the epileptic generators very near the lesions successfully removed in surgery for epilepsy.

Significance: The ICA of ictal EEG events may be a useful add-on to the tools used to establish the connection between epileptic scalp activity and the cortical tubers originating it, in patients with TSC considered for surgery of epilepsy.

(c) 2007 International Federation of Clinical Neurophysiology. Published by Elsevier Ireland Ltd. All rights reserved.
\end{abstract}

Keywords: Epilepsy; Seizure; Tuberous sclerosis complex; ICA; sLORETA

\section{Introduction}

Tuberous sclerosis (TS) is one of the most common neuro-cutaneous syndromes and the patients very often have pharmacoresistant focal epilepsy (Holmes and Stafstrom, 2007). These epilepsies are usually associated with complex and multifocal spike activity in the scalp EEG that leads to the false impression that several brain areas are

\footnotetext{
* Corresponding author. Address: Department of Neurophysiology, Hospital Júlio de Matos, $\mathrm{Av}^{\mathrm{a}}$ do Brasil nr 53, 1749-002 Lisbon, Portugal. Tel.: +351 969851734; fax: +351 217819809 .

E-mail address: a.leal@netcabo.pt (A.J.R. Leal).
}

producing seizure activity and so these patients were formerly not considered for surgery (Jansen et al., 2007). It became apparent in recent years that interictal spikes are a poor predictor of the localization of seizure onset, and many patients have consistent seizure onset foci and can benefit from a surgical procedure, despite having the previous complex interictal pattern (Perot and Weir, 1966; Bebin et al., 1993).

The selection process remains nevertheless difficult because of the complex and variable temporal characteristics of the EEG and ictal behavioral manifestations. In this setting a better understanding of the relationship between ictal and interictal activity becomes important, in order to select wisely the good surgical candidates. 
We propose a strategy of study favoring ictal EEG analysis, combining Independent Component Analysis (ICA) and source analysis, to establish the critical connection between EEG scalp recordings and the underlying brain lesions.

\section{Methods}

Four consecutive patients from the Pediatric Neurology Department of Hospital Dona Estefânia with the diagnosis of tuberous sclerosis (TS) and epilepsy refractory to medical treatment were included in the present study. The patients were being considered for surgery of epilepsy and the neurophysiological and brain imaging data were obtained with this goal in mind.

All patients underwent long-term video-EEG recordings, using 19 (patients 2 and 4) or 27 (10-20 plus $\mathrm{F}_{9 / 10}$, $\mathrm{T}_{9 / 10}, \mathrm{P}_{9 / 10}, \mathrm{Fp}_{\mathrm{z}}$ and $\mathrm{O}_{\mathrm{z}}$, for patients 1 and 3) gold electrodes glued to the scalp with colodium. The sampling rate was $256 \mathrm{~Hz}$ and a band pass of $0.1-70 \mathrm{~Hz}$ was used. Electrode positions were measured using the method of De Munck et al. (1991) for patients 1 and 3 and were obtained from standard positions for the 10-20 system for patients 2 and 4.

The MRIs were obtained using clinical protocols that included in all cases T1 volumetric sequences allowing reconstruction of the brain anatomy and co-registration with the electrodes in the scalp.

The interictal spikes were divided in topographical classes using visual inspection by an experienced clinical neurophysiologist (AL). For each topography a representative spike was selected and used to perform a template based automatic search for similar spikes using the BESA 5.1 software (MEGIS, Graefelfing, Germany). The detected spikes were averaged $(N=15-40)$ and submitted to source analysis.

The ictal recordings were processed (three per patient) using the EEGLAB toolbox (Delorme and Makeig, 2004) and methods detailed elsewhere (Leal et al., 2006). Briefly, the EEG was epoched from -20 to $30 \mathrm{~s}$, with zero time at seizure onset. A decomposition in independent components (ICs) using the Infomax algorithm (Bell and Sejnowski, 1995; Makeig et al., 1997) was performed and the ICs expressing rhythmical activity after seizure onset were selected for source analysis of their spatial component.

Source analysis for the averaged interictal spikes and the selected ictal ICA components was done with the sLORETA software package (Pascual-Marqui, 2002), available at http://www.unizh.ch/keyinst/NewLORETA/

LORETA01.htm.

\section{Clinical data}

The patients included in this study have been studied repeatedly at our department with video-EEG to document uncontrollable partial seizures (Table 1). In all of them we were convinced that a single onset zone for the epileptic fits was present, despite the variable EEG features and clinical manifestations of the seizures along time.

Patient 1 started at an early age ( 2 months) with motor seizures involving the right face and arm, which lasted 13 min and on average occurred 15-20 times daily. The early

Table 1

Clinical and neurophysiological data

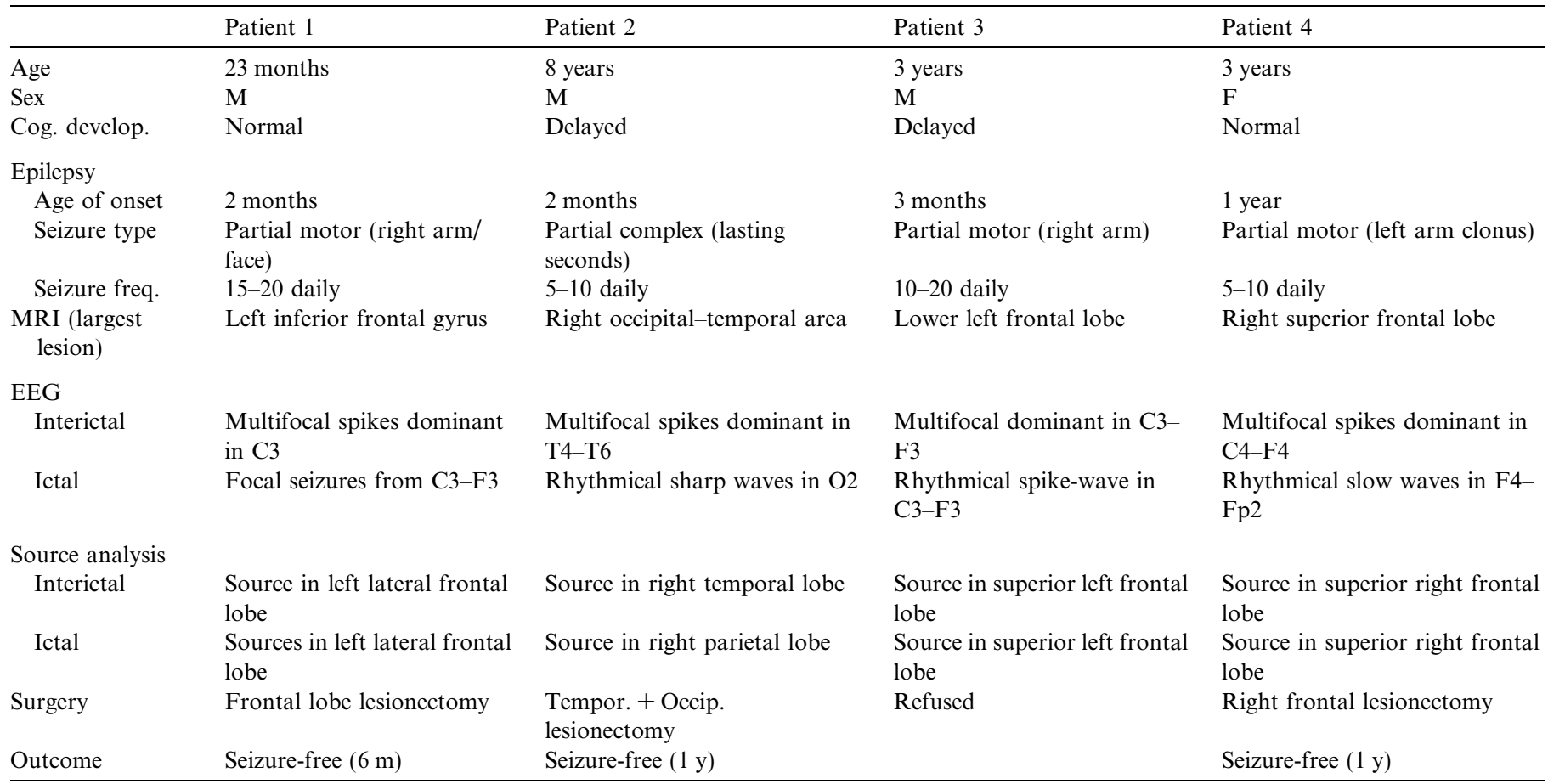


(a)

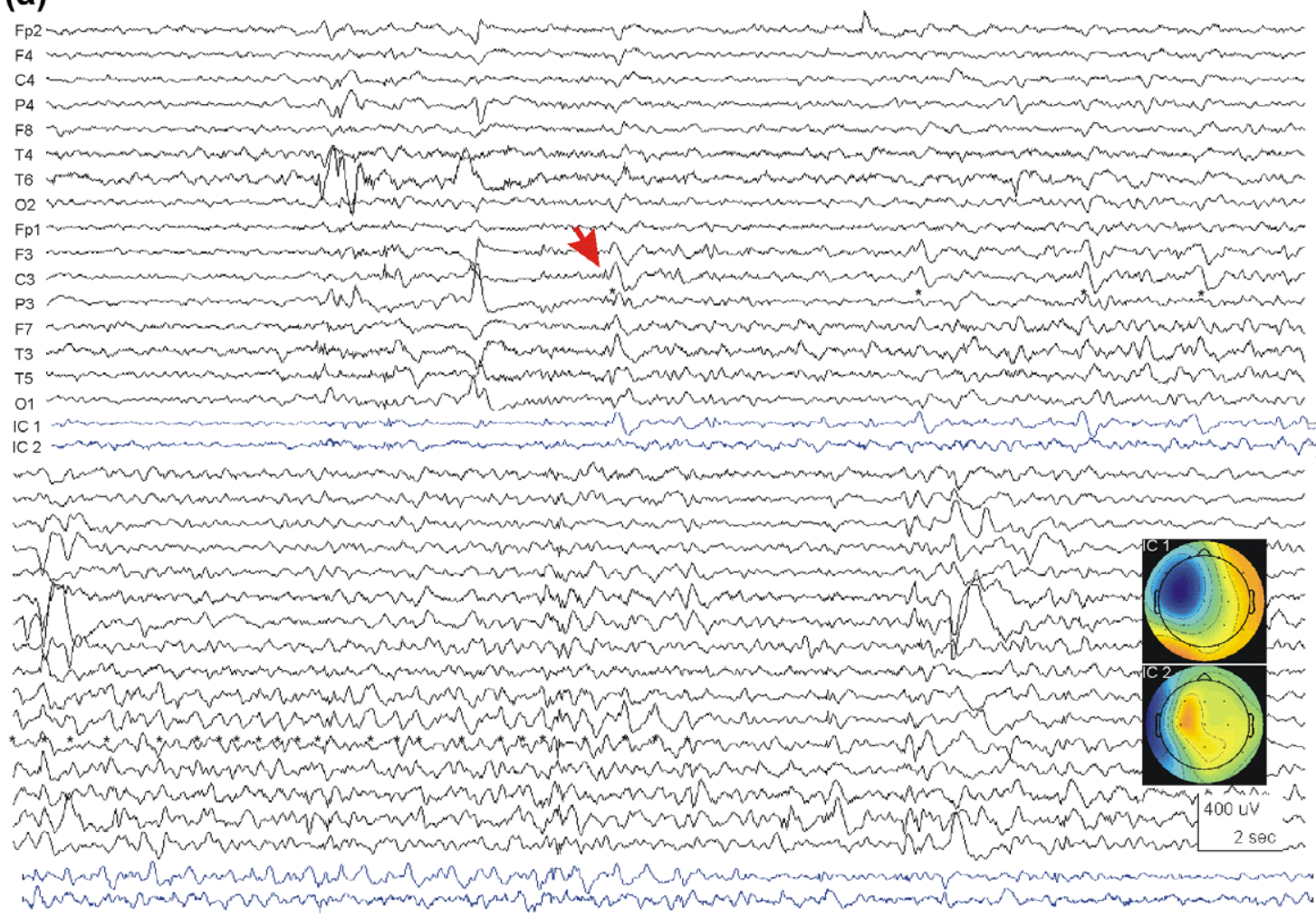

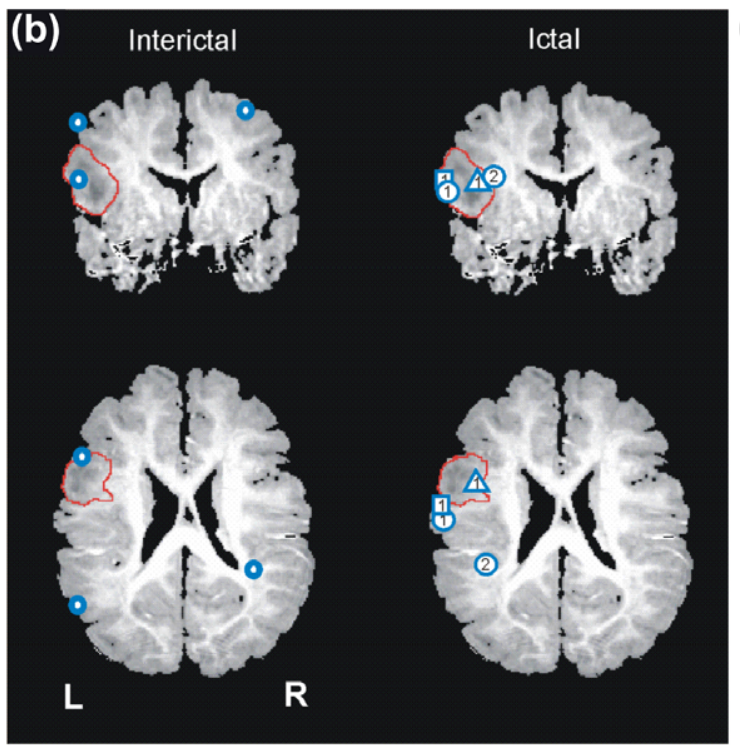

(c)

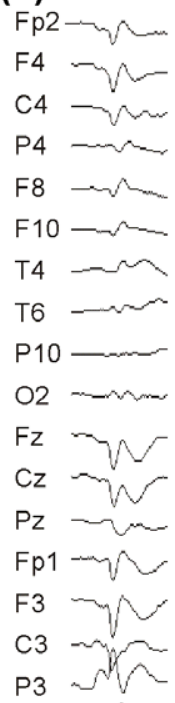

F7

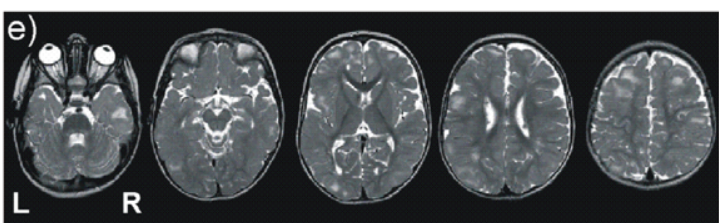

F7

F9

T3

Po

01

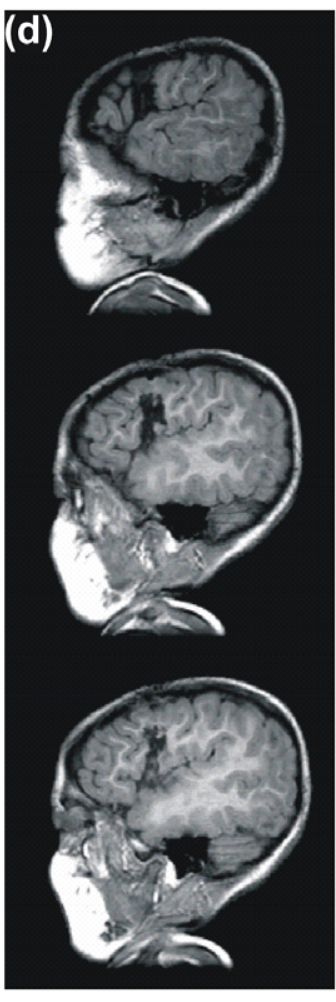

Fig. 1. (a) Seizure event for patient 1, with onset of EEG rhythmical activity in electrodes F3-C3 (arrow and asterisks) and the two ICs demonstrating synchronous activation (IC1 and IC2). In the right is shown the topography of the two ICs, which have a dipolar distribution over the scalp (vertical scale is $400 \mu \mathrm{V}$ and the horizontal scale $2 \mathrm{~s}$ ). (b) Source analysis with the sLORETA method. Projected on the MRI plane with the suspected tuber is represented the interictal maximum for the averaged interictal spikes (circles, left) and for three ictal events (circles, square and triangle on the right). The solutions are localized in the interface between the lesion and normal brain. When more than one IC is present per seizure, they are numbered. (c) Average interictal EEG spikes with the dominant one on the left (horizontal scale $200 \mathrm{~ms}$, vertical scale $100 \mu \mathrm{V}$ ). (d) MRI after successful surgery of epilepsy, demonstrating removal of the tuber. (e) T2 sequence to demonstrate the various tubers in patient 1. 
(a)
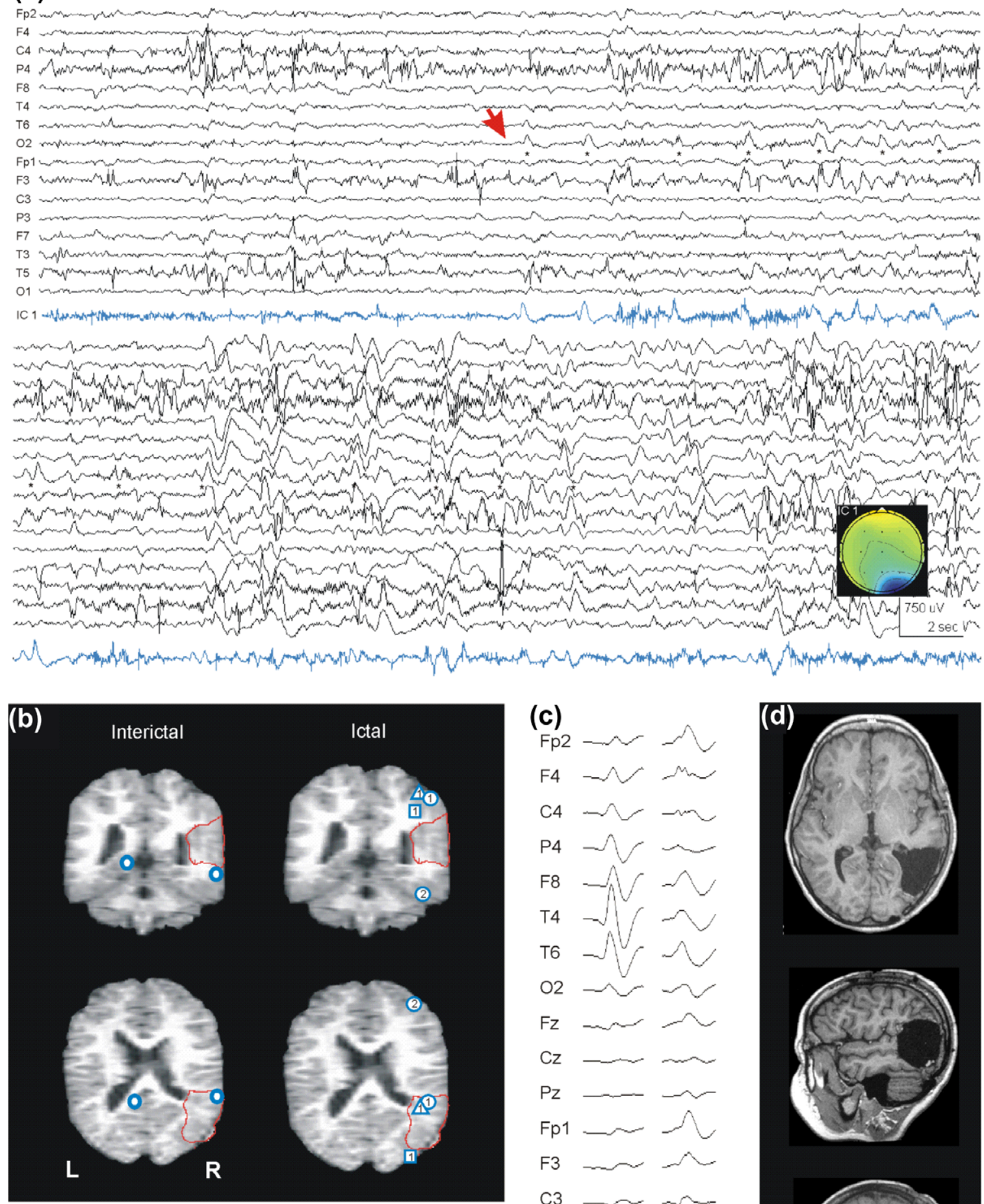

(c)
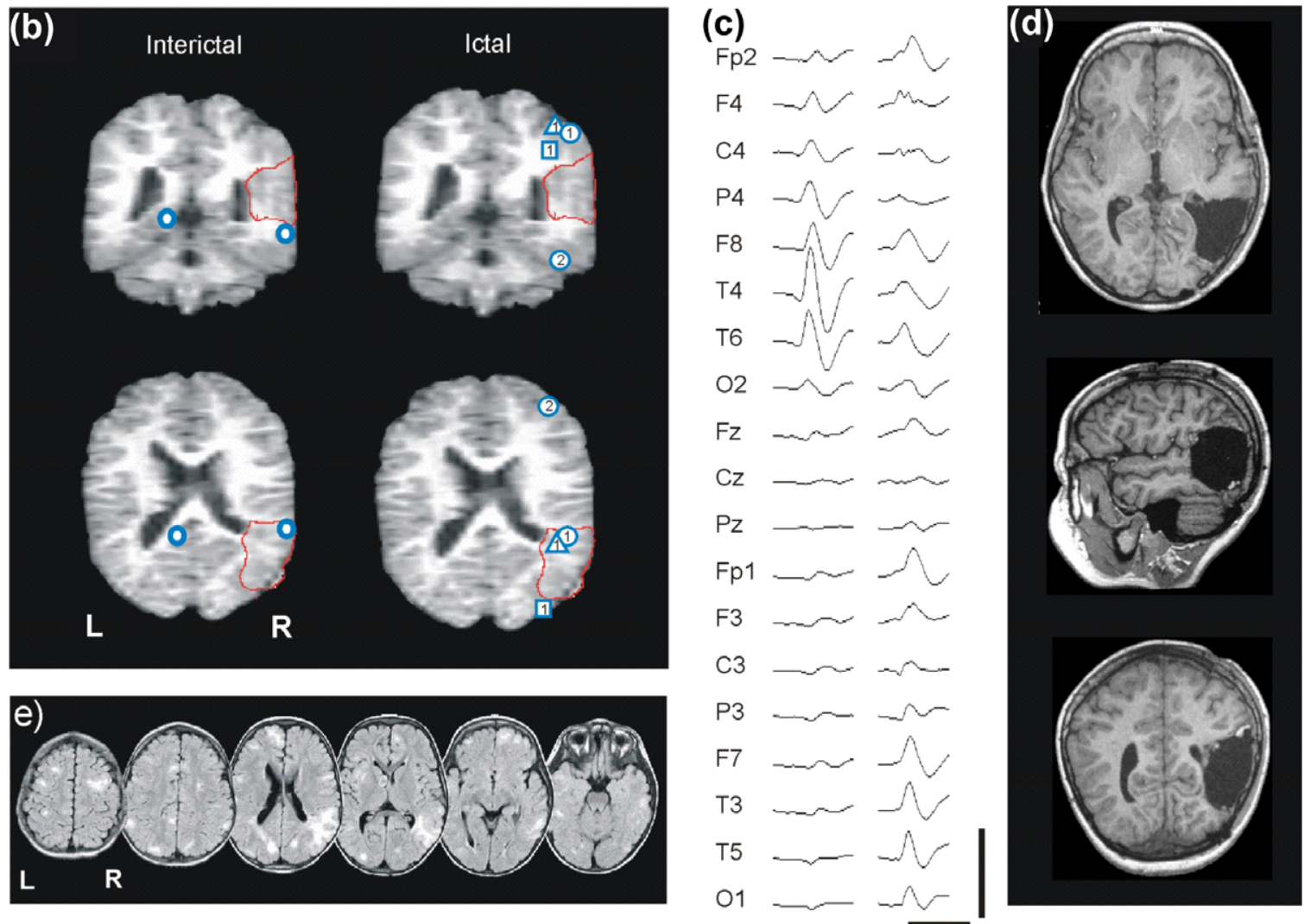

Fig. 2. (a) Seizure event for patient 2, demonstrating onset of rhythmical activity in electrode $\mathrm{O} 2$ (arrow and asterisks). This activity was recovered by a single IC, with maximum over the right posterior areas (below, right) (vertical scale $750 \mu \mathrm{V}$ and the horizontal scale $2 \mathrm{~s}$ ). (b) sLORETA results for the averaged interictal spikes (left) and for the ictal events (right). There is a large spatial difference between the two solutions. (c) Average EEG spike (horizontal scale $300 \mathrm{~ms}$, vertical scale $100 \mu \mathrm{V}$ ). (d) MRI after successful surgery for epilepsy. (e) MRI sequence demonstrating the multiple tubers in this patient. 
video-EEG study at age of 3 months, demonstrated focal interictal spikes with phase reversal on bipolar montages over C3 (Fig. 1c, left) and several seizure events with clear onset in the same electrode (Fig. 1a). Later studies at ages of 8 and 10 months confirmed the same ictal onset, despite a more difficult EEG analysis due to increased low frequency activity and decreased amplitude of the epileptic rhythm. Interictal activity demonstrated multifocal spikes (Fig. 1c, right). The MRI presented a left frontal lesion involving the lower gyrus, which was interpreted as the probable epileptogenic tuber (Fig. 1e).

Patient 2 also had an early onset of epileptic seizures, which consisted of episodes of interruption of consciousness lasting for several seconds mainly in the morning period. They occurred daily and repeatedly, in burst that could last for 1-2 h, and were followed by several hours of confusion. Severe behavioral problems became apparent with attention deficits, lack of social skills and repeated episodes of aggression to his school companions. The repeated evaluation with video-EEG and long-term ambulatory EEG allowed us to identify a consistent ictal rhythm over the right occipital area (Fig. 2a), while the interictal spikes were multifocal, with a more consistent focus over the right temporal electrodes (Fig. 2c, left). The comparison of the ictal activity and the MR imaging implied the large dysplastic lesion over the right temporal and occipital cortex as the originator of the seizures (Fig. 2e).

Patient 3 demonstrated clinical and EEG features very similar to the ones of patient 1 , with early onset of partial motor seizures involving the right arm and associated with ictal onset in the C3-F3 electrodes (Fig. 3a), reproducible in three studies. An associated tuber was found in the lower gyrus of the left frontal lobe (Fig. 3b). The patient's mother refused surgery.

Patient 4 started with partial motor seizures involving the left arm at the age of 1 year. The episodes occurred 5-10 times daily and were associated with a fast EEG ictal rhythm with onset in the right frontal lobe (Fig. 4a). The interictal activity was multifocal but more consistent in the frontal lobe electrodes (Fig. 4c, left). Several small tubers could be identified over the right frontal lobe in the MRI (Fig. 4e).

Patient 1 was operated at our hospital, while patients 2 and 4 were operated at another institution.

After surgery all patients remain seizure-free. In patient 1 there was a clear improvement in the cognitive development and communication skills. Patient 2 demonstrated a striking reduction in the behavioral disturbances, with improved attention and social integration. In patient 4 no problems in behavior were detected either before or after surgery.

\section{Results}

All patients expressed multifocal interictal spikes in the scalp EEG, but a clearly dominant type (the most frequent one) was always present, with consistent morphology and dipolar topography (Figs. 1c, 2c, 3c and 4c, left). For most patients (1,3 and 4) there was lobar concordance between the most dysplastic brain area and the localization of the phase reversal of the dominant spikes in bipolar montages. The sources obtained from these averaged interictal spikes were localized in the lobe with the largest tuber for all patients with the maximum sLORETA statistic score near the interface between the dysplastic and normal brain tissue (Figs. 1b, 2b, 3b and 4b). Other spike topographies were associated with very distinct localizations for their generators (Figs. 1b, 2b, 3b and 4b), which overall provide little consistency in defining the epileptic brain area in a given patient from interictal recordings.

The ictal paroxysms showed a very similar topographic pattern to the one of the dominant interictal spikes in patients 3 and 4 (Figs. $3 b$ and $4 b$ ), but a different topography in the remaining patients (Figs. 1b and $2 b$ ). The scalp distribution of seizure activity remained stable in different seizures and the ictal semiology was compatible with the lobar localization of the EEG rhythmic activity (Table 1).

The decomposition of the ictal EEG by ICA produced one or two rhythmic components synchronous with the raw scalp signal (Figs. 1a, 2a, 3a and 4a). The sources obtained from these ICs demonstrated a close spatial relationship with the largest tubers in all four patients (Figs. $1 \mathrm{~b}, \mathrm{c} ; 2 \mathrm{~b}, \mathrm{c} ; 3 \mathrm{~b}, \mathrm{c}$ and $4 \mathrm{~b}, \mathrm{c})$. These ictal sources showed spatial consistency in different seizure events.

In the three cases successfully submitted to surgery the interictal sources were localized in the neighbourhood but outside the resection volume (Figs. 1d, 2d and 4d) and at the interface between the normal and most dysplastic area for the patient not operated. The ictal sources were also predominantly located at the interface between the most dysplastic brain areas and the normal brain. The ECoG performed before cortical resection in patient 1 revealed abundant spikes in the brain surrounding the tuber, but not in the gyrus containing it. This suggests that the lesion is not an intrinsic generator of interictal spikes, which more likely originate in the adjacent cortex.

\section{Discussion}

The main conclusion of our work is that the source analysis of ictal rhythms in patients with epilepsy associated with TS produces consistent results in different seizure events, with a close spatial relationship with the brain area removed in successful surgery for epilepsy. This contrasts with the complex and multifocal interictal spike activity typical of these patients, which makes it very difficult to build a consistent picture of the epileptic activity dynamics from this type of recordings. In some cases, such as in our case 2 , there is a significant spatial separation between ictal and interictal sources, which makes it unreliable to extrapolate which brain area is originating the seizures from interictal activity alone. 
(a)
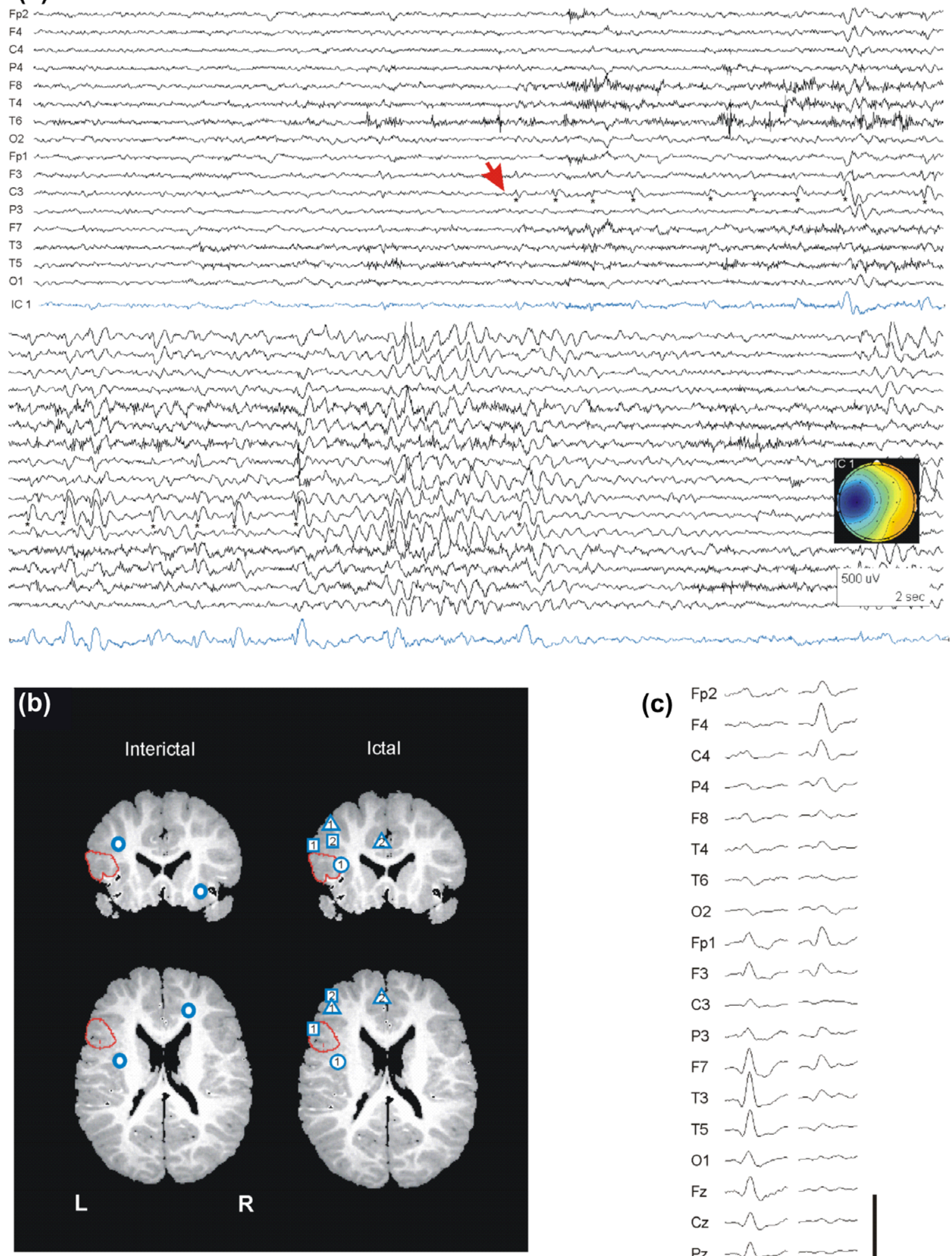

(c)

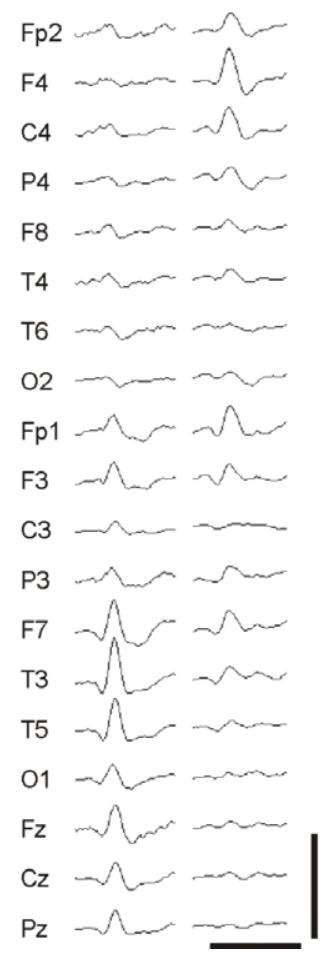

Fig. 3. (a) Seizure event for patient 3, demonstrating onset of rhythmical activity in electrode C3 (arrow). This activity was recovered by a single IC (bellow, right) (vertical scale $500 \mu \mathrm{V}$ and the horizontal scale $2 \mathrm{~s}$ ). (b) sLORETA results for the averaged interictal spikes (left) and for the ictal events (right). There is partial spatial overlap of the generators for the two types of epileptic activity. (c) Average EEG spikes (scales as in Fig. 2).

In clinical neurophysiology the analysis of ictal EEG, which is of fundamental importance for the selection of patients for surgery of epilepsy, has lagged behind the analysis of the interictal spikes (Jansen et al., 2006; Iida et al., 
(a)
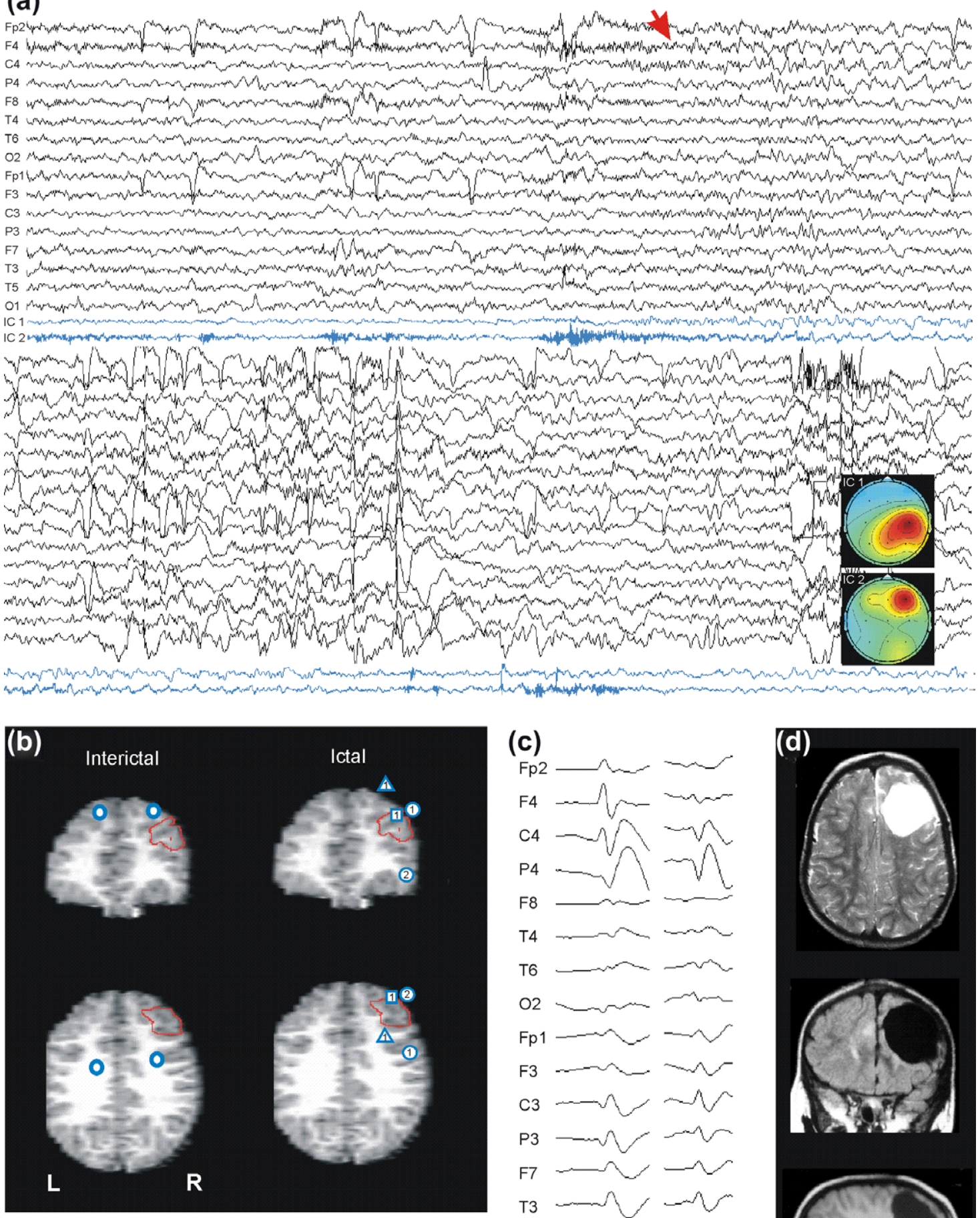

(c)

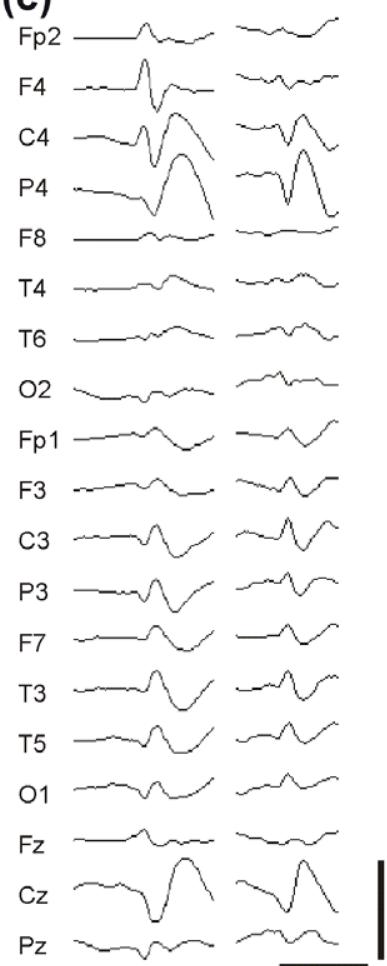

(d)
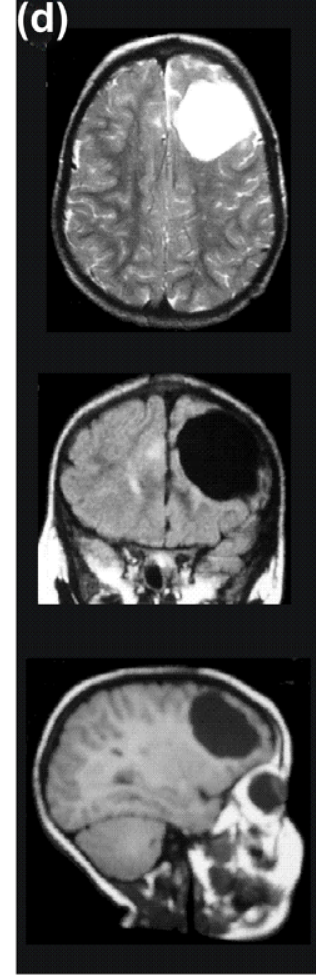

Fig. 4. (a) Seizure event for patient 4, demonstrating onset of rhythmical activity in electrode F4 (arrow). This activity was recovered by two ICs, with maximum over the right frontal lobe (bellow, right) (vertical scale $750 \mu \mathrm{V}$ and the horizontal scale $2 \mathrm{~s}$ ). (b) sLORETA results for the averaged interictal spikes (left) and for the ictal events (right). (c) Average EEG spikes (scales as in Fig. 2). (d) MRI after successful surgery for epilepsy. (e) MRI sequence demonstrating the multiple tubers in the frontal lobe of this patient. 
2005; Kamimura et al., 2006; Sperli et al., 2006) mainly due to the poorer signal to noise ratio (SNR). Several additional factors contribute to this, but the more important ones are the movement and EMG artifacts induced by the ictal behavioral manifestations and also the dynamic character of the epileptic activity which spreads to various brain areas using complex and poorly understood pathways. Furthermore the usual methods of improving the SNR such as averaging and frequency filtering are not very effective in this setting. The introduction of blind source separation methods such as ICA (Makeig et al., 1997) and spatial filtering offers a much more promising way to improve the SNR, which is a requirement for the use of more advanced methods of analysis.

The power of ICA decomposition of the EEG to identify and remove movement artifacts is well established (Makeig et al., 1997; Urrestarazu et al., 2004) and has been confirmed in our study. The high amplitude and temporal evolution of such components makes their identification easy and their removal allows a more detailed inspection of seizure activity. In our patients the observation of the artifactfree EEG revealed that the rhythmic activity present in the seizure was also present in the remaining ICs. In order to study the ICs specifically related to the ictal events we selected the rhythmical components demonstrating synchronous activity with the rhythms in the raw EEG (Figs. 1a, 2a, 3a and 4a). This procedure reduced the ictal data to a restricted number of ICs (1 or 2), whose temporal pattern of activation matched the rhythms that in the original EEG were widespread in the scalp, producing a significant convergence of information. The fact that the scalp maps of these ICs have dipolar topography further suggests that focal and fixed sources may be good models for these generators of interest.

The ICA provides a robust method to overcome the two main limitations of ictal analysis: it allows separation and selective elimination of artifacts which in general have very distinct temporal dynamics from the intrinsic epileptic activity; and also separates the contribution of epileptic activity from distinct brain areas which lead to significant volume conductor mixing in the scalp potentials. Previous attempts to do source analysis of ictal events with spatiotemporal dipoles restricted the study to seizures with few artifacts and good signal to noise ratio, such as temporal lobe seizures (Assaf and Ebersole, 1997). Lantz et al. (2001) attempted to address more representative neocortical epilepsies using microstate segmentation of the EEG to obtain snapshots of the intracranial generators throughout the event. This method does not correct for the problem of volume conductor mixing of concomitant activation of different sources which might be present (such as in the events of Figs. 1a and 4a) and is potentially misleading.

In order to gain insight into the intracranial localization of the generators of the IC patterns seen in the scalp, we adopted the sLORETA algorithm (Pascual-Marqui, 2002), who has good localizing capabilities for focal sources (Wagner et al., 2004). The method provides a smooth distribution of statistical scores in the volume space, with a maximum at the source localization. Anatomical constraints to the volume space can be used, for example restricting the solution space to the cortical volume as in our study, and this further improves the localization of sources while remaining compatible with the physiology.

The interictal spike activity in our patients is multifocal, but in all a dominant topography could be identified which produced sources in the neighbourhood of the epileptogenic lesions. This agrees with the results of Seri et al. (1998), which also have been able to recover sources near frontal lobe tubers in cases of TSC and frontal lobe spikes. Nevertheless, in 2 out of 4 patients there was a discrepant topography and corresponding source localization between the two types of epileptic activity, with results suggesting that the ictal analysis leads to new and spatially more consistent information as compared to the analysis of interictal spikes only.

The surgical results in our cases were good and give support to the suggestion from previous studies implicating the most dysplastic brain areas as the usual area of origin of ictal activity (Bebin et al., 1993). Two major obstacles stand in the way to surgery in these patients: one is the usually complex and variable interictal spike activity that seldom points unambiguously to a particular brain area; the other is the change in the ictal behavioral manifestations along time due not to new epileptic foci but to different propagation patterns of the epileptic activity from the epileptic focus. In our opinion achieving a good case selection for surgery is critically dependent on demonstrating a consistent pattern of ictal activity in the EEG in several seizures and eventually at different ages. Only then we can be confident that a single focus might provide the explanation to the epilepsy of a given patient. In this strategy the convergence of information offered by ICA, complemented with source analysis techniques, seems a promising tool in the pre-surgical study of patients with TS and epilepsy.

\section{Acknowledgements}

The authors are grateful to Daniel Borges, Adilia Seabra, Daniel Carvalho, Elisabete Lage and Rita Pinto for technical support and to Dr. Jan the Munck for comments on the manuscript.

\section{References}

Assaf B, Ebersole J. Continuous source imaging of scalp ictal rhythms in temporal lobe epilepsy. Epilepsia 1997;38(10):1114-23.

Bebin EM, Kelly PJ, Gomez MR. Surgical treatment for epilepsy in cerebral Tuberosis Sclerosis. Epilepsia 1993;34(4):651-7.

Bell AJ, Sejnowski TJ. An information maximisation approach to blind separation and blind deconvolution. Neural Comput 1995;7:1129-59.

De Munck JC, Vijn PC, Spekreijse H. A practical method for determining electrode positions on the head. Electroencephalogr Clin Neurophysiol 1991;78(1):85-7.

Delorme A, Makeig S. EEGLAB: an open source toolbox for analysis of single-trial EEG dynamics including independent component analysis. J Neurosci Methods 2004;134(1):9-21. 
Holmes GL, Stafstrom CE. The Tuberosis Sclerosis Study Group. Tuberosis sclerosis complex and epilepsy: recent developments and future challenges. Epilepsia 2007;48(4):617-30.

Iida K, Otsubo H, Mohamed I, Okuda C, Ochi A, Weiss S, et al Characterizing magnetoencephalographic spike sources in children with tuberous sclerosis complex. Epilepsia 2005;46(9):1510-7.

Jansen F, Huffelen A, Algra A, Nieuwenhuizen O. Epilepsy surgery in tuberous sclerosis: a systematic review. Epilepsia 2007;48(8):1477-84.

Jansen F, Huiskamp G, Huffelen A, Bourez-Swart M, Boere E, Gebbink $\mathrm{T}$, et al. Identification of the epileptogenic tuber in patients with tuberous sclerosis: a comparison of high-resolution EEG and MEG. Epilepsia 2006;47(1):108-14.

Kamimura T, Tohyama J, Oishi M, Akasaka N, Kanazawa O, Sasagawa $\mathrm{M}$, et al. Magnetoencephalography in patients with tuberous sclerosis and localization-related epilepsy. Epilepsia 2006;47(6):991-7.

Lantz G, Michel CM, Seeck M, Blanke O, Spinelli L, Thut G, et al. Spaceoriented segmentation and 3-dimensional source reconstruction of ictal EEG patterns. Clin Neurophysiol 2001;112:688-97.

Leal A, Dias A, Vieira JP. Analysis of the EEG dynamics of epileptic activity in gelastic seizures using decomposition in independent components. Clin Neurophysiol 2006;117:1595-601.
Makeig S, Jung TP, Bell AJ, Ghahremani D, Sejnowski TJ. Blind separation of auditory event-related brain responses into independent components. Proc Natl Acad Sci USA 1997;94(20):10979-84.

Perot P, Weir B. Tuberous sclerosis: surgical therapy for seizures. Arch Neurol 1966;15:498-506.

Pascual-Marqui RD. Standardized low resolution brain electromagnetic tomography (sLORETA): technical details. Methods Findings Exp Clin Pharmacol 2002;24D:5-12.

Seri S, Cerquiglini A, Pisani F, Michel C, Pascual-Marqui R, Curatolo P. Frontal lobe epilepsy associated with tuberous sclerosis: electroencephalographic-magnetic resonance imaging fusioning. J Child Neurol 1998;13(1):33-8.

Sperli F, Spinelli L, Seeck M, Kurian M, Michel C, Lantz G. EEG source imaging in pediatric epilepsy surgery: a new perspective in presurgical workup. Epilepsia 2006;47(6):981-90.

Urrestarazu E, Iriarte J, Alegre M, Valencia M, Viteri C, Artieda J. Independent component analysis artifacts in ictal recordings. Epilepsia 2004;45(9):1071-8.

Wagner M, Fuchs M, Kastner J. Evaluation of sLORETA in the presence of noise and multiple sources. Brain Topogr 2004;16(4):277-80. 